\title{
Ilaria Vitali (dir.), Intrangers
}

\section{Carminella Biondi}

\section{OpenEdition}

\section{Journals}

Édition électronique

URL : http://journals.openedition.org/studifrancesi/3888

DOI : 10.4000/studifrancesi.3888

ISSN : 2421-5856

\section{Éditeur}

Rosenberg \& Sellier

\section{Édition imprimée}

Date de publication : 1 décembre 2012

Pagination : 617-618

ISSN : 0039-2944

\section{Référence électronique}

Carminella Biondi, « Ilaria Vitali (dir.), Intrangers », Studi Francesi [En ligne], 168 (LVI | III) | 2012, mis en ligne le 30 novembre 2015, consulté le 08 mars 2021. URL : http://journals.openedition.org/ studifrancesi/3888 ; DOI : https://doi.org/10.4000/studifrancesi.3888

Ce document a été généré automatiquement le 8 mars 2021.

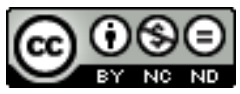

Studi Francesi è distribuita con Licenza Creative Commons Attribuzione - Non commerciale - Non opere derivate 4.0 Internazionale. 


\title{
Ilaria Vitali (dir.), Intrangers
}

\author{
Carminella Biondi
}

\section{RÉFÉRENCE}

ILARIA VITALI (dir.), Intrangers, Bruxelles, Academia/L'Harmattan, 2011. Tome I, Postmigration et nouvelles frontières de la littérature beur, pp. 147; tome II: Littérature beur, de l'écriture à la traduction, pp. 189.

1 «L'Intranger, c'est un mot que j'ai inventé que si tu es pas d'origine difficile tu peux pas piger, mais moi je t'explique, ça veut juste dire que tu es un étranger dans ton propre pays, mais ne me demande pas si le pays en question c'est l'Algérie ou la France». (tome I, p.5) En exergue à ce recueil d'essais, cette citation tirée du roman Allah superstar d'Y.B. donne au lecteur une clef d'interprétation fondamentale et trace le parcours à suivre. Publié sous la direction d'Ilaria Vitali, docteur de l'Université de Bologne et de l'Université Paris IV-Sorbonne, Intrangers propose de reconsidérer l'ensemble de la littérature beur à trente ans de sa naissance.

2 Divisé en deux petits tomes pour faciliter la lecture et la réception, cet ouvrage collectif recueille une douzaine d'articles de belle facture, qui comportent une véritable mine d'informations sur la littérature beur et ses alentours. Si le premier tome (Postmigration et nouvelles frontières de la littérature beur) porte sur la naissance et les évolutions de la littérature en question, le second (Littérature beur, de l'écriture à la traduction), se penche, pour la première fois de manière systématique, sur ses aspects langagiers et traductologiques. Chaque tome s'ouvre sur une introduction de l'auteur, qui ne se borne pas à proposer une excellente synthèse des évolutions de cette littérature des années 1980 à nos jours, mais fournit plusieurs clefs d'analyse et des pistes interprétatives innovatrices. "À presque trente ans de la naissance de cette littérature de la post-migration, la question du classement demeure centrale et tout critique s'approchant de ces auteurs aura affaire in primis aux problèmes de définition qui les accompagnent» (tome I, p. 9). Les chercheurs qui s'occupent de littérature beur le savent bien: il est très difficile - voire illusoire - de classer des auteurs qui sont par 
définition inclassables, le risque majeur étant celui d'établir des étiquettes imprécises ou arbitraires et, qui plus est, ghettoïsantes. Ilaria Vitali assume ce débat comme point de départ, en dé-construisant les étiquettes qui se sont succédé au fil du temps, de la littérature «franco-maghrébine» à la littérature «de banlieue», en passant par la littérature «urbaine». Elle propose, pour sa part, la notion de «nébuleuse beur», en entendant par là une constellation d'auteurs qui se rapprochent par les thèmes et les motifs traités, par le style et le langage mis en œuvre, parfois même par les expériences vécues, mais qui peuvent avoir des paraboles existentielles et des parcours d'écriture très différents. Une étiquette qui ne serait donc pas une boîte étanche, mais plutôt un tiers espace ouvert, comme le dirait Homi Bhabha, s'organisant grâce au jeu incessant d'interactions, de transferts, d'échanges et de réorientations multiples entre l'ici et l'ailleurs. Voilà donc que la «trouvaille identitaire» d'Y.B. prend tout son sens: «Ce néologisme, intranger» affirme Vitali «me semble saisir mieux que d'autres le concept d'une identité interstitielle, qui se bâtit à partir des assonances et des dissonances de deux cultures différentes que l'on s'efforce de mettre au diapason» (tome I, p. 13).

La prégnance de ce débat terminologique nous introduit au contenu du premier tome de cet ouvrage, consacré aux aspects sociologiques et à l'influence de la «médiasphère» dans la naissance et l'évolution de la littérature dite «beur». Si l'article d'ouverture de Kathryn KLEPPINGER (tome I, pp. 21-46) analyse avec finesse les premières apparitions médiatiques des écrivains en question - Mehdi Charef entre autres - pour montrer leurs changements de posture par rapport aux medias, Robert VARGA (tome I, pp. 71-89) se penche sur l'affaire du «faux beur» Paul Smaïl-et sur sa polémique hypermédiatisée avec Azouz Begag - pour interroger l'identité de «l'auteur beur» et la remettre en question. Laura REECK (tome I, pp. 47-70) nous montre, quant à elle, un autre aspect intéressant qui concerne la «médiasphère»: par le biais de l'analyse du film documentaire Sur ma ligne que l'écrivain Rachid Djaïdani a tourné pendant l'écriture de son roman Mon nerf, elle nous fait percevoir une véritable osmose entre les différents medias expressifs, une osmose que l'auteur met en œuvre en même temps qu'il opère sa dé-construction de l'étiquette d'artiste «de banlieue». Les deux derniers articles de ce premier tome, respectivement de Mireille LE BRETON (tome I, pp. 93-117) et d'Elisabetta QUARTA (tome I, pp. 119-139), proposent de sortir des grilles interprétatives abusées et invitent à lire les textes de la «nébuleuse beur» par le biais de nouvelles pistes d'analyse, en tenant compte des ouvrages - romans aussi bien qu'essais-les plus récents.

4 Le second tome d'Intrangers s'ouvre sur une seconde présentation du directeur de l'ouvrage, qui focalise ici son regard sur les innovations stylistiques et langagières de la littérature en question. «Si l'on observe de plus près le champ littéraire contemporain, l'un des changements les plus radicaux par rapport aux ouvrages des années 1980 concerne le traitement stylistique des textes et l'exploitation du langage. Les auteurs contemporains ont exaspéré l'usage des emprunts, des xénismes, le goût du verlan, de l'argot, voire de la cyberl@ngue» (tome II, p. 9-10). Les deux premiers articles de ce tome sont en effet focalisés sur le renouveau de la littérature en question: Stève PUIG (tome II, pp. 21-46) observe le passage de la "génération beur» à celle des artistes «urbains», en registrant une évolution au niveau aussi bien stylistique que thématique; Abdelbaki ALLAOUI (tome II, pp. 47-73) montre, quant à lui, de quelle manière Rachid Djaïdani a su s'approprier la filmographie sur la banlieue pour ensuite en réinventer le langage. 
5 Force est de souligner que cet ouvrage est aussi le premier qui consacre un espace considérable aux enjeux traductologiques des romans qui font partie de la constellation beur. Trois articles de ce deuxième tome sont effectivement consacrés aux défis traductologiques de quelques romans-clef parmi les plus récents: si Alena POLICKÁ et Caroline FIÉVET (tome II, pp.77-122) se penchent sur une analyse minutieuse des xénismes dans le roman Kiffe kiffe demain de Faïza Guène, Emmanuelle ERTEL (tome II, pp. 123-154) examine, elle, les traductions anglaise et américaine du même roman pour en relever les différences. L'article d'Ilaria viTALI (tome II, pp. 155-184), traductrice de plusieurs écrivains de la "post-migration», clôt cet ouvrage par l'analyse des défis concernant la traduction des romans de Rachid Djaïdani dans le contexte italien.

6 Reste à savoir si le mot «beur» disparaîtra un jour ou deviendra dans l'univers francophone un umbrella term, tel que «black» l'est devenu dans le monde anglo-saxon. Limitons-nous à dire, avec Ilaria Vitali, que «si le phénomène littéraire beur a suscité autant d'intérêt et de débats en France et ailleurs, c'est qu'il constitue un corpus massif à l'intérieur de ces écritures de deuxième génération et qu'il a forgé, bon gré mal gré, sa propre esthétique» (tome II, p. 17).

7 Un ouvrage très riche, novateur et bien documenté, qui ne saurait manquer dans la bibliothèque des chercheurs en littératures francophones. 